\title{
Fast PageRank Computation via a Sparse Linear System
}

\author{
Gianna M. Del Corso, Antonio Gulli, and Francesco Romani
}

Abstract. Recently, the research community has devoted increased attention to reducing the computational time needed by web ranking algorithms. In particular, many techniques have been proposed to speed up the well-known PageRank algorithm used by Google. This interest is motivated by two dominant factors: (1) the web graph has huge dimensions and is subject to dramatic updates in terms of nodes and links, therefore the PageRank assignment tends to became obsolete very soon; (2) many PageRank vectors need to be computed according to different choices of the personalization vectors or when adopting strategies of collusion detection.

In this paper, we show how the PageRank computation in the original random surfer model can be transformed in the problem of computing the solution of a sparse linear system. The sparsity of the obtained linear system makes it possible to exploit the effectiveness of the Markov chain index reordering to speed up the PageRank computation. In particular, we rearrange the system matrix according to several permutations, and we apply different scalar and block iterative methods to solve smaller linear systems. We tested our approaches on web graphs crawled from the net. The largest one contains about 24 millions nodes and more than 100 million links. Upon this web graph, the cost for computing the PageRank is reduced by $65 \%$ in terms of Mflops and by $92 \%$ in terms of time respect to the power method commonly used.

\section{Introduction}

The research community has devoted increased attention to reducing the computation time needed by web ranking algorithms. In fact, the web changes very rapidly: in one week more than $25 \%$ of links are changed and $5 \%$ of "new content" is created [Cho and Roy 04]. This result indicates that search engines need

(C) A K Peters, Ltd.

I542-795I/05 \$0.50 per page 
to update link based ranking metrics very often and that a week-old ranking may not reflect very well the current importance of the pages.

Many efforts have been devoted to improve PageRank [Brin and Page 98, Page et al. 98], the well-known ranking algorithm used by Google. The core of Page-Rank exploits an iterative weight assignment of ranks to the web pages, until a fixed point is reached. This fixed point turns out to be the (dominant) eigenpair of a matrix derived from the web graph itself. Brin and Page originally suggested to compute this pair using the well-known power method [Golub and Van Loan 96], and they also gave a nice interpretation of PageRank in terms of Markov chains. Recent studies about PageRank address at least two different needs: first, the desire to reduce the time spent weighting the nodes of the web graph, which takes several days of computation, and second, the need to assign many PageRank values to each web page. This is necessary for PageRank's personalization [Haveliwala 02, Haveliwala et al. 03, Jeh and Widom 03] that was recently presented by Google as beta-service (see http://labs.google. com/personalized/) or for some heuristic for collusion-proof PageRank [Zhang et al. 04] algorithms that requires the computation of many different PageRank vectors for different choices of a parameter.

Previous approaches followed different directions such as the attempt to compress the web graph to fit it into main memory [Boldi and Vigna 04] or the implementation in external memory of the algorithms [Haveliwala 99, Chen et al. 02]. A very interesting research track exploits efficient numerical methods to reduce the computation time. These kinds of numerical techniques are the most promising, and we have seen many intriguing results in the last few years that accelerate the power iterations [Kamvar et al. 03a, Haveliwala 99, Lee et al. 03]. In the literature [Arasu et al. 02, Lee et al. 03, Page et al. 98] models are presented that treat in a different way pages with no out-links. In this paper we consider the original PageRank model (see Section 3), and by using numerical techniques, we show that this problem can be transformed in an equivalent linear system of equations, where the coefficient matrix is as sparse as the web graph itself. This new formulation of the problem makes it natural to investigate the structure of the sparse coefficient matrix in order to exploit its reducibility. Moreover, since many numerical iterative methods for linear system solution can benefit by a reordering of the coefficient matrix, we rearrange the matrix, increasing the data locality and reducing the number of iterations needed by the solving methods (see Section 5). In particular, we evaluate the effect of many different permutations, and we apply several methods, such as power, Jacobi, Gauss-Seidel, and Reverse Gauss-Seidel [Varga 62], on each of the rearranged matrices. The disclosed structure of the permuted matrix makes it possible to use block methods that turn out to be more powerful than the scalar ones. Note 
that the phase of reordering a matrix according to a given permutation requires extra computational effort, but the time spent reordering the matrix is negligible with respect to the time required to solve the system. A more important consideration is that the same reordering can be used to solve many PageRank problems with different personalization vectors.

We tested our approaches on a web graph crawled from the net of about 24 million nodes and more than 100 million links. Our best result, achieved by a block method, is a reduction of $65 \%$ in Mflops and of $92 \%$ in time with the respect of the power method taken as the reference method to compute the PageRank vector.

\section{Definitions and Notations}

In this section we give some notations and definitions that will be useful in the rest of the paper. Let $M$ by an $n \times n$ matrix. A scalar $\lambda$ and a nonzero vector $\mathbf{x}$ are an eigenvalue and the corresponding (right) eigenvector of $M$ if $M \mathbf{x}=\lambda \mathbf{x}$. In the same way, if $\mathbf{x}^{T} M=\lambda \mathbf{x}, \mathbf{x}$ is called the left eigenvector corresponding to the eigenvalue $\lambda$. Note that, a left eigenvector is a (right) eigenvector of the transpose matrix. A matrix is row-stochastic if its rows are nonnegative and the sum of each row is one. In this case, it is easy to show that there exists a dominant eigenvalue equal to 1 and a corresponding eigenvector $\mathbf{x}=(c, c, \ldots, c)^{T}$, for any constant $c$. A very simple method for the computation of the dominant eigenpair is the power method [Golub and Van Loan 96], which, for stochastic irreducible matrices, is convergent for any nonnegative starting vector. A stochastic matrix $M$ can be viewed as a transition matrix associated to a family of Markov chains, where each entry $M_{i j}$ represents the probability of a transition from state $i$ to state $j$. By the Ergodic Theorem for Markov chains [Stewart 95], an irreducible stochastic matrix $M$ has a unique steady state distribution, that is, a vector $\pi$ such that $\pi^{T} M=\pi^{T}$. This means that the stationary distribution of a Markov chain can be determined by computing the left eigenvector of the stochastic matrix $M$. Given a graph $G=(V, E)$ and its adjacency matrix $A$, let $\operatorname{outdeg}(i)$ be the out-degree of vertex $i$ that is the number of nonzeros in the $i$ th row of $A$. A node with no out-links is called dangling.

\section{Google's PageRank Model}

In this section we review the original idea of Google's PageRank [Brin and Page 98]. The web is viewed as a directed graph (the web graph) $G=(V, E)$, 
where each of the $N$ pages is a node and each hyperlink is an edge. The intuition behind this model is that a page $i \in V$ is important if it is pointed by other pages which are in turn important. This definition suggests an iterative fixed-point computation to assigning a rank of importance to each page in the web. Formally, in the original model [Page et al. 98], a random surfer sitting on the page $i$ can jump with equal probability $p_{i j}=1 / \operatorname{outdeg}(i)$ to each page $j$ adjacent to $i$. The iterative equation for the computation of the PageRank vector $\mathbf{z}$ becomes $z_{i}=\sum_{j \in I_{i}} p_{j i} z_{j}$, where $I_{i}$ is the set of nodes in-linking to the node $i$. The component $z_{i}$ is the "ideal" PageRank of page $i$ and is given by the sum of the PageRanks assigned to the nodes pointing to $i$, weighted by the transition probability $p_{i j}$. The equilibrium distribution of each state represents the ratio between the number of times the random walk is in the state over the total number of transitions, assuming that the random walk continues for infinite time. In matrix notation, the above equation is equivalent to the solution of the following system of equations: $\mathbf{z}^{T}=\mathbf{z}^{T} P$, where $P_{i j}=p_{i j}$. This means that the PageRank vector $\mathbf{z}$ is the left eigenvector of $P$ corresponding to the eigenvalue 1. In the rest of the paper, we assume that $\|\mathbf{z}\|_{1}=\sum_{i=1}^{N} z_{i}=1$, since most of the time one is not interested in assigning an exact value to each $z_{i}$ but rather in the relative rank between the nodes.

The "ideal" model unfortunately has two problems. The first one is due to the presence of dangling nodes, which capture the surfer indefinitely. Formally, a dangling node corresponds to an all-zero row in $P$. As a consequence, $P$ is not stochastic and the Ergodic Theorem cannot be applied. A convenient solution to the problem of dangling nodes is to define a matrix $\bar{P}=P+D$, where $D$ is the rank-one matrix defined as $D=\mathbf{d v}^{T}$, and $d_{i}=1$ iff $\operatorname{outdeg}(i)=0$. The vector $\mathbf{v}$ is a personalization vector that records a generic surfer's preference for each page in $V$ [Haveliwala 02, Jeh and Widom 03]. The matrix $\bar{P}$ imposes a random jump to every other page in $V$ whenever a dangling node is reached. Note that the new matrix $\bar{P}$ is stochastic. In Section 4 , we refer to this model as the natural model and compare it with other approaches proposed in the literature. The second problem with the "ideal" model is that the surfer can "get trapped" by a cyclic path in the web graph. Brin and Page [Brin and Page 98] suggest to enforce irreducibility by adding a new set of artificial transitions that with low probability jump to all nodes. Mathematically, this corresponds to defining a matrix $\widehat{P}$ as

$$
\widehat{P}=\alpha \bar{P}+(1-\alpha) \mathbf{e v}^{T}
$$

where $\mathbf{e}$ is the vector with all entries equal to 1 and $\alpha$ is a constant, $0<\alpha<1$. At each step, with probability $\alpha$ the random surfer follows the transitions described by $\bar{P}$, while with probability $(1-\alpha)$ she/he bothers to follows links and jumps to 
any other node in $V$ accordingly to the personalization vector $\mathbf{v}$. The matrix $\widehat{P}$ is stochastic and irreducible, and both these conditions imply that the PageRank vector $\mathbf{z}$ is the unique steady state distribution of the matrix $\widehat{P}$ such that

$$
\mathbf{z}^{T} \widehat{P}=\mathbf{z}^{T} .
$$

From (3.1) it turns out that the matrix $\widehat{P}$ is explicitly

$$
\widehat{P}=\alpha\left(P+\mathbf{d} \mathbf{v}^{T}\right)+(1-\alpha) \mathbf{e v}^{T} .
$$

The most common numerical method used to solve the eigenproblem (3.2) is the power method [Golub and Van Loan 96]. Since $\widehat{P}$ is a rank-one modification of $\alpha P$, it is possible to implement a power method that multiplies only the sparse matrix $P$ by a vector and upgrades the intermediate result with a constant vector at each step, as suggested by Haveliwala in [Haveliwala 99].

The eigenproblem (3.2) can be rewritten as a linear system. By substituting (3.3) into (3.2) we get $\mathbf{z}^{T}\left(\alpha P+\alpha \mathbf{d} \mathbf{v}^{T}\right)+(1-\alpha) \mathbf{z}^{T} \mathbf{e v}=\mathbf{z}^{T}$, which means that the problem is equivalent to the solution of the linear system of equations

$$
S \mathbf{z}=(1-\alpha) \mathbf{v}
$$

where $S=I-\alpha P^{T}-\alpha \mathbf{v} \mathbf{d}^{T}$, and we make use of the fact that $\mathbf{z}^{T} \mathbf{e}=\sum_{i=1}^{N} z_{i}=1$. The transformation of the eigenproblem (3.2) into (3.4) opens the route to a large variety of numerical methods not completely investigated in the literature. In the next section we present a lightweight solution to handling the nonsparsity of $S$.

\section{A Sparse Linear System Formulation}

In this section we show how we can compute the PageRank vector as the solution of a sparse linear system. We remark that the way one handles the dangling node is crucial, since there can be a huge number of them. According to Kamvar et al. [Kamvar et al. 03b], a 2001 sample of the web containing 290 million pages had only 70 million nondangling nodes. This large amount of nodes without out-links includes both pages that do not point to any other page and also pages whose existence is inferred by hyperlinks but not yet reached by the crawler. Besides, a dangling node can represent a pdf, ps, txt, or any other file format gathered by a crawler but with no hyperlinks pointing outside. Page et al. [Page et al. 98] adopted the drastic solution of completely removing the dangling nodes. In this way, the size of the problem is sensibly reduced, but a large amount of information present in the web is ignored. This has an impact on both the dangling 
(a)

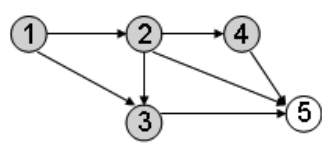

(c) (b)

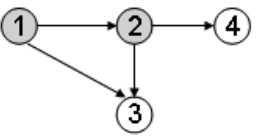

(d)

(1) $\longrightarrow$ (2)

(1)

Figure I. Removing the dangling node in (a) generates new dangling nodes, which are in turn removed in (b), (c), and (d). At the end of the process, no node receives a PageRank assignment.

nodes - which are simply not ranked - and on the remaining nodes - which don't take into account the contribution induced by the random jump from the set of dangling nodes. Moreover, removing this set of nodes could potentially create new dangling nodes, which must in turn be removed (see Figure 1). Therefore, the nodes with an assigned PageRank could be a fraction of the web graph.

Arasu et al. [Arasu et al. 02] handled dangling nodes in a different way with respect to the natural model presented in Section 3. They modify the web graph by imposing that every dangling node has a self loop. In terms of matrices, $\bar{P}=P+F$ where $F_{i j}=1$ iff $i=j$ and $\operatorname{outdeg}(i)=0$. The matrix $\bar{P}$ is row stochastic, and the computation of PageRank is solved using a random jump similar to equation (3.3), where the matrix $F$ replaces $D$. This model is different from the natural model, as is evident from the following example.

Example 4.I. Consider the graph and the associated transition matrix in Figure 2. The PageRank obtained, by using the natural model, orders the nodes as $(2,3,5,4,1)$. Arasu's model orders the nodes as $(5,4,2,3,1)$. Note that in the latter case node 5 ranks better than node 2 , which is not what one expects.

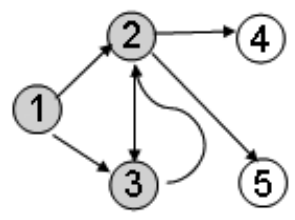

$$
P=\left(\begin{array}{ccccc}
0 & 1 / 2 & 1 / 2 & 0 & 0 \\
0 & 0 & 1 / 3 & 1 / 3 & 1 / 3 \\
0 & 1 & 0 & 0 & 0 \\
0 & 0 & 0 & 0 & 0 \\
0 & 0 & 0 & 0 & 0
\end{array}\right)
$$

Figure 2. An example of a graph whose rank assignment differs if the dangling nodes are treated as in the model presented by Arasu et al. in [Arasu et al. 02]. 
From the above observations we believe that it is important to take into account the dangling nodes, and we consider the natural model, which better captures the behavior of a random surfer. The dense structure of the matrix $S$ poses serious problems to the solution of the linear system (3.4). For this reason, the problem has been largely addressed as an eigenproblem, while we have seen very few attempts to solve the problem as a linear system. Computing the PageRank vector with the power method allows one to exploit the sparsity of the matrix $P$. In fact, it is common to implement the power method in such a way that the matrix-vector multiplications involve only the sparse matrix $P$ while the rank-one modifications are handled separately [Haveliwala 99].

In the following, we show how to manage dangling nodes in a direct and lightweight manner that makes it possible to use iterative methods for linear systems. In particular, we prove formally the equivalence of (3.4) to the solution of a system involving only the sparse matrix $R=I-\alpha P^{T}$. The next theorem makes use of a very powerful tool: the Sherman-Morrison formula (see [Golub and Van Loan 96], paragraph 2.1.3). It is well known that the Sherman-Morrison formula is unstable; however, we use it here only as an elegant technique for proving the theorem without any need of implementing it.

Theorem 4.2. The PageRank vector $\mathbf{z}$ solution of (3.4) is obtained by solving the system $R \mathbf{y}=\mathbf{v}$ and taking $\mathbf{z}=\mathbf{y} /\|\mathbf{y}\|_{1}$.

Proof. Since $S=R-\alpha \mathbf{v} \mathbf{d}^{T}$, equation (3.4) becomes $\left(R-\alpha \mathbf{v} \mathbf{d}^{T}\right) \mathbf{z}=(1-\alpha) \mathbf{v}$, that is, a system of equations where the coefficient matrix is the sum of a matrix $R$ and a rank-one matrix. Note that $R$ is nonsingular since $\alpha<1$ and therefore all the eigenvalues of $R$ are different from zero. We can use the Sherman-Morrison formula (see [Golub and Van Loan 96], paragraph 2.1.3) to compute the inverse of the rank-one modification of $R$. As a consequence, we have

$$
\left(R-\alpha \mathbf{v} \mathbf{d}^{T}\right)^{-1}=R^{-1}+\frac{R^{-1} \mathbf{v d}^{T} R^{-1}}{1 / \alpha+\mathbf{d}^{T} R^{-1} \mathbf{v}} .
$$

From (4.1), denoting by $\mathbf{y}$ the solution of the system $R \mathbf{y}=\mathbf{v}$, we have

$$
\mathbf{z}=(1-\alpha)\left(1+\frac{\mathbf{d}^{T} \mathbf{y}}{1 / \alpha+\mathbf{d}^{T} \mathbf{y}}\right) \mathbf{y}
$$

which means that $\mathbf{z}=\gamma \mathbf{y}$, and the constant $\gamma=(1-\alpha)\left(1+\frac{\mathbf{d}^{T} \mathbf{y}}{1 / \alpha+\mathbf{d}^{T} \mathbf{y}}\right)$ can be computed normalizing $\mathbf{y}$ in such a way that $\|\mathbf{z}\|_{1}=1$.

In summary, we have shown that, in order to compute the PageRank vector $\mathbf{z}$, we can solve the system $R \mathbf{y}=\mathbf{v}$ and then normalize $\mathbf{y}$ to obtain the PageRank 
vector $\mathbf{z}$. This means that the rank-one matrix $D$ in the PageRank model which accounts for the dangling pages plays a role only in the scaling factor $\gamma$. Moreover, the computation of $\gamma$ is not always necessary, and this step can occasionally be omitted.

Note that the matrix used by Arasu and al. [Arasu et al. 02] is also sparse due to the way in which they deal with the dangling nodes, but the PageRanks obtained don't rank the nodes in a natural way (see Example 4.1). Instead, our approach guarantees a more natural ranking and handles the density of $S$ by transforming a dense problem into one that uses the sparse matrix $R$.

Bianchini et al. in [Bianchini et al. 03] prove that the iterative method derived by (3.2) and involving $\widehat{P}$ produces the same sequence of vectors as the Jacobi method applied to matrix $R$. Recently Eiron et al. [Eiron et al. 04] proposed another way to deal with dangling nodes. They assign separately a rank to dangling and nondangling pages, and their algorithm requires the knowledge of a complete strongly-connected subgraph of the web.

\section{I. The Conditioning of the Problem in the New Formulation}

When solving a linear system, one must devote particular attention to the conditioning of the problem, the magic number accounting for the "hardness" of solving a linear system. The condition number of a matrix $A$ is defined as $\operatorname{cond}(A)=\|A\|\left\|A^{-1}\right\|$ for any matrix norm. It is easy to show, as proved by Kamvar and Haveliwala [Kamvar and Haveliwala 03], that the condition number in the 1-norm of $S$ is $\operatorname{cond}(S)=\frac{1+\alpha}{1-\alpha}$, which means that the problem tends to become ill-conditioned as $\alpha$ goes to one. For the conditioning of $R$, we can prove the following theorem.

Theorem 4.3. The condition numbers expressed in the 1-norm of matrices $S$ and $R$ are such that

$$
\operatorname{cond}(R) \leq \operatorname{cond}(S)
$$

Moreover, the inequality is strict if and only if from every node there is a direct path to a dangling node.

Proof. In order to prove the theorem, we have to show that $\operatorname{cond}(R) \leq \frac{1+\alpha}{1-\alpha}$. We have

$$
\|R\|_{1}=\max _{j=1, \ldots, n} \sum_{i=1}^{n}\left|r_{i j}\right|=\max _{j=1, \ldots, n}\left(1+\alpha \sum_{i=1, i \neq j}^{n} p_{j i}\right) .
$$

Then, if $P \neq O,\|R\|_{1}=1+\alpha$. Note that $R^{-1}$ is a nonnegative matrix, hence

$$
\left\|R^{-1}\right\|_{1}=\left\|\mathbf{e}^{T} R^{-1}\right\|_{1}=\left\|R^{-T} \mathbf{e}\right\|_{\infty} .
$$


Moreover,

$$
R^{-T}=(I-\alpha P)^{-1}=\sum_{i=0}^{\infty} \alpha^{i} P^{i} .
$$

Since every entry of the vector $P \mathbf{e}$ is less or equal 1 , we have $P^{i} \mathbf{e} \leq \mathbf{e}$, hence

$$
\left\|R^{-1}\right\|_{1}=\left\|\sum_{i=0}^{\infty} \alpha^{i} P^{i} \mathbf{e}\right\| \leq \sum_{i=0}^{\infty} \alpha^{i}=\frac{1}{1-\alpha},
$$

which proves that $\operatorname{cond}(R) \leq \operatorname{cond}(S)$.

Let us now prove with Markov chain state transitions that the inequality is strict if from every page it is possible to reach a dangling node. Since $P$ has dangling nodes, $P$ is reducible. Let $k, k \geq 1$, be the number of stronglyconnected components of the web graph. We can permute rows and columns of $P$, grouping together the nodes belonging to the same connected component and listing the dangling nodes in the last rows. Therefore, $P$ can be expressed in the reduced form

$$
P=\left[\begin{array}{ccccc}
P_{11} & P_{1,2} & \cdots & \cdots & P_{1, k} \\
& P_{2,2} & \cdots & & P_{2, k} \\
& & \ddots & & \vdots \\
& & & P_{k-1, k-1} & P_{k-1, k} \\
O & & & & O
\end{array}\right]
$$

where the diagonal blocks $P_{i i}$ are irreducible. By hypothesis, from every web page we can reach a dangling node with a finite number of clicks. In terms of the matrix $P$, this means that, for every $i, 1 \leq i \leq k$, each block $P_{i i}$ has at least one row whose sum is strictly less than 1 . An extension of the Gershgorin circle Theorem [Varga 62, Theorem 1.7, page 20] ensures that the spectral radius of every diagonal block $P_{i i}$ is less than 1 . Since the eigenvalues of $P$ are the eigenvalues of the diagonal blocks, this guarantees that $\rho(P)<1$. Let us consider the iterative method $\mathbf{x}_{i+1}=P \mathbf{x}_{i}$; since $\rho(P)<1$, this method is convergent to the zero vector, for every choice of the starting vector $\mathbf{x}_{0}$. Then, choosing $\mathbf{x}_{0}=\mathbf{e}$, there exists an integer $i$ such that $P^{i} \mathbf{e}<\mathbf{e}$. From (4.2) we have

$$
\left\|R^{-T}\right\|_{1}=\left\|\sum_{i=0}^{\infty} \alpha^{i} P^{i} \mathbf{e}\right\|_{\infty}<\left\|\sum_{i=0}^{\infty} \alpha^{i} \mathbf{e}\right\|=\frac{1}{1-\alpha},
$$

which proves the "if" part. To prove the "only if" part, assume by contradiction that there is at least one page from which it is not possible to reach a dangling page, and assume that this page belongs to the $h$ th connected component. Since $P_{h h}$ is irreducible, this means that from every node in $P_{h h}$, it is not possible 
to reach a dangling page. Hence, there is at least one index $i, 1 \leq i \leq k-1$, such that the strip corresponding to the $i$ th block is zero, except for the diagonal block $P_{i i}$. This means that the diagonal block $P_{i i}$ has an eigenvalue equal to one, hence $\rho(P)=1$ and $P_{i i} \mathbf{e}_{i}=\mathbf{e}_{i}$, which implies that $\operatorname{cond}(R)=(1+\alpha) /(1-\alpha)$.

Theorem 4.3 proves that the condition number of matrix $R$ is always less than or equal to the condition number of $S$. This means that computing the PageRank vector that solves the system with matrix $R$ is never worst than computing the one that solves the system involving $S$. In fact, the system in $R$ is less sensitive to errors due to the finite representation of the numbers appearing in $P$ than the system involving $S$ is.

Note that if the condition that guarantees the strict inequality between the condition number of $S$ and $R$ is not satisfied, there exists a reordering of $R$ which allows one to split the original problem into two disjoint subproblems. As we will see in Section 5, this is computationally convenient, and moreover, the conditioning of the two subproblems should be compared with the conditioning of the two subproblems regarding $S$.

\section{Exploiting the Web Matrix Permutations}

In Section 4 we have shown how to transform the linear system involving the matrix $S$ into an equivalent linear system, where the coefficient matrix $R$ is as sparse as the web graph. The new sparse formulation allows one to exploit the effectiveness of other iterative procedures to compute the PageRank vectors, which were not applicable when dealing with $S$. To solve the linear system $R \mathbf{y}=\mathbf{v}$, two convenient strategies to use are the Jacobi and Gauss-Seidel methods [Varga 62], because they use space comparable to that used by the power method. These methods are convergent if and only if the spectral radius of the iteration matrix is strictly lower than one. Moreover, the rate of convergence depends on the spectral radius, and the lower the radius is, the faster the convergence. Since $R=I-\alpha P$ where $P$ is a nonnegative matrix, $R$ is a so-called $M$-matrix, and it is well known that both the Jacobi and Gauss-Seidel methods are convergent and that the Gauss-Seidel method applied to $M$-matrices is always faster than the Jacobi method [Varga 62].

When solving a sparse linear system, a common practice [Douglas et al. 00] is to look for a reordering scheme that reduces the (semi)bandwidth for increasing data locality and hence the time spent for each iteration. Moreover, if the matrix is reducible, the problem can be split into smaller linear systems that can be solved in cascade. 
The simpler permutation scheme is the one that separates dangling from nondangling pages. In this case the matrix system involving $R$ has a very simple shape,

$$
\left[\begin{array}{cc}
I-\alpha P_{1}^{T} & O \\
-\alpha P_{2}^{T} & I
\end{array}\right]\left[\begin{array}{l}
\mathbf{y}_{1} \\
\mathbf{y}_{2}
\end{array}\right]=\left[\begin{array}{l}
\mathbf{v}_{1} \\
\mathbf{v}_{2}
\end{array}\right]
$$

and once the system $\left(I-\alpha P_{1}^{T}\right) \mathbf{y}_{1}=\mathbf{v}_{1}$ is solved, the vector $\mathbf{y}_{2}$ is computed with only a matrix vector multiplication as $\mathbf{y}_{2}=\mathbf{v}_{2}+\alpha P_{2}^{T} \mathbf{y}_{1}$. Two recent papers, one by Eiron et al. [Eiron et al. 04] and the other by Langville and Meyer [Langville and Meyer 04], arrive, with different reasoning, to the same formulation of the problem, observing that a problem much smaller than the initial one has to be solved. The problem involving $P_{1}$ is still solved using the power method, and they did not further investigate other reordering schemes that could increase the benefits of permutation strategies.

Note that once $R$ is reordered into a block triangular matrix, a natural way to solve the system is to use forward/backward block substitution. For instance, on the lower block triangular system

$$
\left[\begin{array}{cccc}
R_{11} & & & \\
R_{21} & R_{11} & & \\
\vdots & & \ddots & \\
R_{m 1} & \cdots & & R_{m m}
\end{array}\right]\left[\begin{array}{c}
\mathbf{y}_{1} \\
\mathbf{y}_{2} \\
\vdots \\
\mathbf{y}_{m}
\end{array}\right]=\left[\begin{array}{c}
\mathbf{v}_{1} \\
\mathbf{v}_{2} \\
\vdots \\
\mathbf{v}_{m}
\end{array}\right]
$$

the solution can be computed as follows:

$$
\left\{\begin{array}{l}
\mathbf{y}_{1}=R_{11}^{-1} \mathbf{v}_{1}, \\
\mathbf{y}_{i}=R_{i i}^{-1}\left(\mathbf{v}_{i}-\sum_{j=1}^{i-1} R_{i j} \mathbf{y}_{j}\right) \quad \text { for } i=2, \ldots, m .
\end{array}\right.
$$

This requires the solution of $m$ smaller linear systems, where the coefficient matrices are the diagonal blocks in the order that they appear. Note that this strategy is always better than applying an iterative method to the whole matrix.

Moreover, for some iterative methods, it may happen that a permutation in the matrix reduces the spectral radius of the iteration matrix and hence the number of iterations needed to reach convergence. This is not the case for the Jacobi method since the spectral radius of the iteration matrix is invariant under permutation. In the same way, the rate of convergence of the power method is also independent of matrix reordering, since it depends only on the spectral properties of the matrix and on the starting vector. A very nice attempt in this direction is given in [Kamvar et al. 03b] where it is shown how reordering the matrix by sorting the URLs lexicographically may help to construct a better starting vector for the power method and to improve data locality. 


\begin{tabular}{|c|c|c|}
\hline Full & Lower Block Triangular & Upper Block Triangular \\
\hline $\mathcal{T}$ & $\mathcal{T} \mathcal{B}$ & $\mathcal{B T}$ \\
$\mathcal{O}_{\mathrm{d}} \mathcal{T}$ & $\mathcal{O}_{\mathrm{d}} \mathcal{T} \mathcal{B}$ & $\mathcal{O}_{\mathrm{d}} \mathcal{B} \mathcal{T}$ \\
$\mathcal{O}_{\mathrm{a}} \mathcal{T}$ & $\mathcal{O}_{\mathrm{a}} \mathcal{T} \mathcal{B}$ & $\mathcal{O}_{\mathrm{a}} \mathcal{B \mathcal { T }}$ \\
$\mathcal{I}_{\mathrm{d}} \mathcal{T}$ & $\mathcal{I}_{\mathrm{d}} \mathcal{T} \mathcal{B}$ & $\mathcal{I}_{\mathrm{d}} \mathcal{B \mathcal { T }}$ \\
$\mathcal{I}_{\mathrm{a}} \mathcal{T}$ & $\mathcal{I}_{\mathrm{a}} \mathcal{T} \mathcal{B}$ & $\mathcal{I}_{\mathrm{a}} \mathcal{B} \mathcal{T}$ \\
\hline
\end{tabular}

Figure 3. Web matrix permutation taxonomy.

A more challenging perspective is the reordering of the web matrix for the Gauss-Seidel method, where opportune permutations can lead both to an increase in data locality and to an iteration matrix with a reduced spectral radius.

The permutation strategies that we propose have two different purposes. The first goal is to increase data locality and decrease, when possible, the spectral radius of the iteration matrix. The second one is to discover a block triangular structure in the web matrix in order to apply block methods as described in equation (5.2).

Applying a permutation to a sparse matrix as well as finding the permutation that satisfies some desiderata is a costly operation. However, it is often convenient to spend more effort to decrease the running time of the solving method when the same matrix is used many times to solve different PageRank problems, as required for personalized web search [Haveliwala 02] or in the case of the heuristic for collusion-proof PageRank [Zhang et al. 04].

The permutations that we considered are obtained by combining different elementary operations. A very effective reordering scheme, denoted by $\mathcal{B}$, is the one obtained by permuting the nodes of the web graph according to the order induced by a BFS visit. The BFS visit makes it possible to discover reducibility of the web matrix, since this visit assigns contiguous permutation indices to pages pointed to by the same source. Therefore, this permutation produces lower block triangular matrices. It has been observed by Cuthill and McKee [Cuthill and McKee 69] that the BFS strategy for reordering sparse symmetric matrices produces a reduced bandwidth when the children of each node are inserted in order of decreasing degree. For this reason, even if $P$ is not symmetric, we examine other reordering schemes that are obtained by sorting the nodes in terms of their degree. In particular we consider the permutation that reorders the pages by decreasing out-degree, denoting this scheme as $\mathcal{O}_{\mathrm{d}}$ while the permutation $\mathcal{O}_{\mathrm{a}}$ sorts the pages of the web matrix by ascending out-degree. Note that these permutations list the dangling pages in the last and in the first rows of the web matrix, respectively. We experimented also with the permutations obtained by 


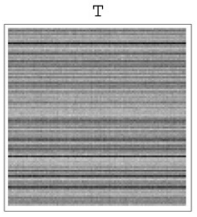

TB

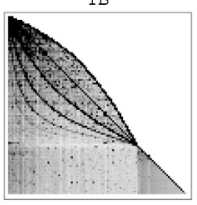

BT

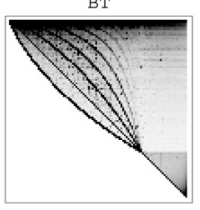

OdT

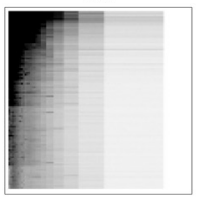

OdTB

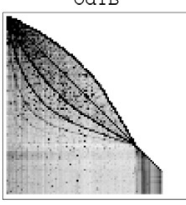

OdBT

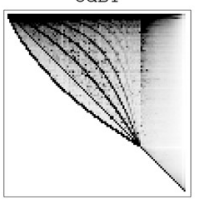

OaT

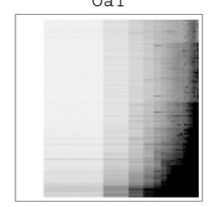

ОаTB

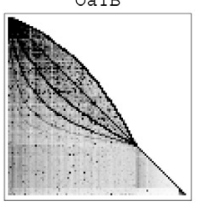

OaBT

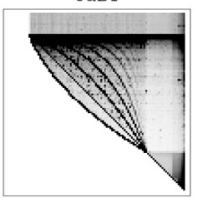

$\operatorname{IdT}$

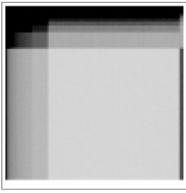

IdTB

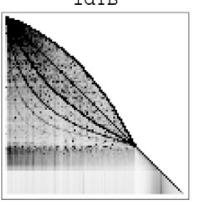

IdBT

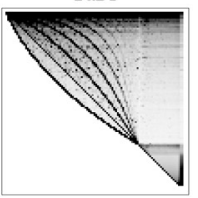

IaT

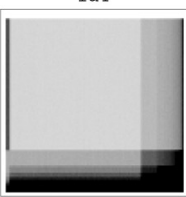

Іатв

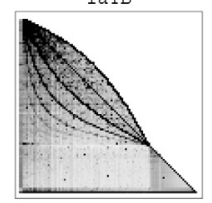

IaBT

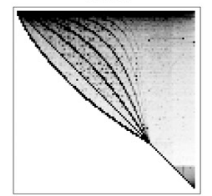

Figure 4. Different shapes obtained by rearranging the web matrix $P$ in accordance to the taxonomy. The first row represents full matrices, the second and third lower and upper block triangular matrices, respectively. The web graph is made of 24 million nodes and 100 million links.

reordering the matrix by ascending and descending in-degree, denoted by $\mathcal{I}_{\mathrm{a}}$ and $\mathcal{I}_{\mathrm{d}}$, respectively. Since $R=I-\alpha P^{T}$, our algorithms needs to compute the transpose of the web matrix; we denote this operation by $\mathcal{T}$. The various operations can be combined to obtain different reorderings of the web matrix as shown in Figure 3. In accordance with the taxonomy in Figure 3, we denote, for instance, $R_{\mathcal{I}_{\mathrm{d}} \mathcal{T} \mathcal{B}}=I-\alpha \Pi(P)$, where the permuted matrix $\Pi(P)$ is obtained by first applying the $\mathcal{I}_{\mathrm{d}}$ permutation, then transposing the matrix, and finally applying the $\mathcal{B}$ permutation on the reordered matrix. The first column in Figure 3 gives rise to full matrices, while the second and third columns produce block triangular matrices due to the BFS's order of visit.

In Figure 4, we show a plot of the structure of a web matrix rearranged according to each item of the taxonomy in Figure 3. It is important to observe that on nonsymmetric matrices the BFS order of visit transforms the matrix into a lower block triangular form, with a number of diagonal blocks that is greater than or equal to the number of strongly-connected components. However, the number of diagonal blocks detected with a BFS depends very much on the starting node of the visit. For example, starting from node 1 in Figure 5, we detect just one component, while starting from node 4 we get four separate components. 


$$
R_{1}=\left(\begin{array}{cccc}
1 & -\alpha & 0 & 0 \\
0 & 1 & -\alpha & 0 \\
0 & 0 & 1 & -\alpha \\
0 & 0 & 0 & 1
\end{array}\right) \quad R_{4}=\left(\begin{array}{cccc}
1 & 0 & 0 & 0 \\
-\alpha & 1 & 0 & 0 \\
0 & -\alpha & 1 & 0 \\
0 & 0 & -\alpha & 1
\end{array}\right)
$$

Figure 5. An example of a connected graph. Applying a BFS visit starting with node 1 , one detects just one component and $R_{1}$ is in unreduced form; starting with node 4 , we get four different components and $R_{4}$ is fully reduced.

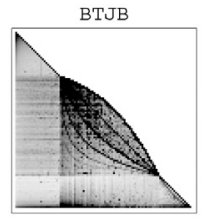

BTB

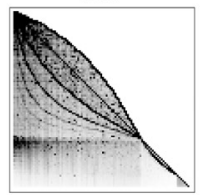

TBJB

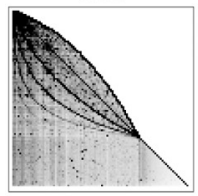

BJBT

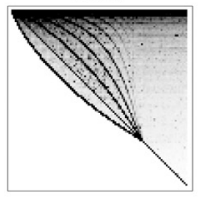

OdBTJB

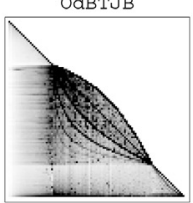

OdBTB

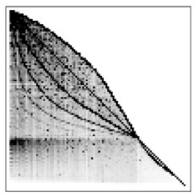

OdTBJB

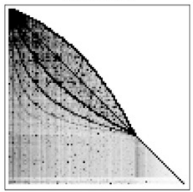

OdBJBT

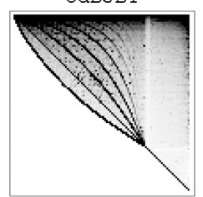

OaBTJB

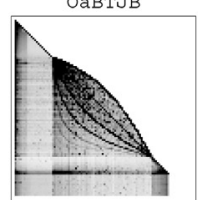

OaBTB

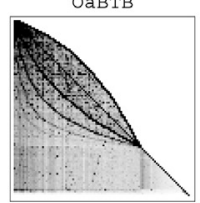

OaTBJB

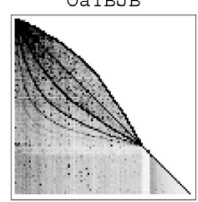

OABJBT

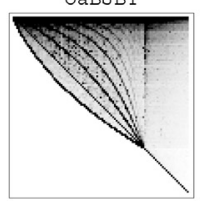

IdBTJB

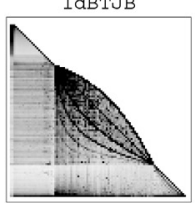

IdBTB

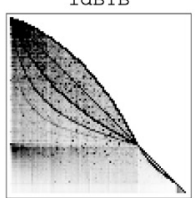

IdTBJB

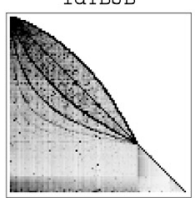

IdBJBT

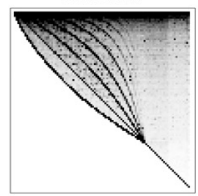

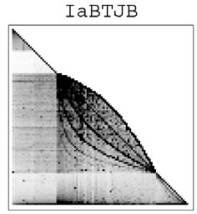

IaBTB

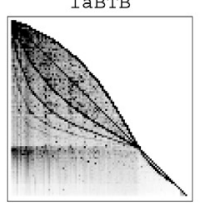

IaTBJB

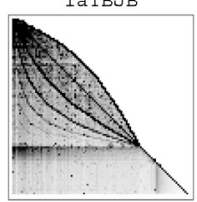

IaBJBT

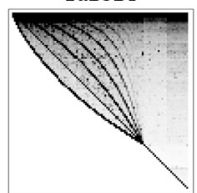

Figure 6. Different shapes obtained by rearranging the web matrix $P$ made of 24 million nodes and 100 million links according to permutations involving two BFS visits. 


\begin{tabular}{|c|c|}
\hline Scalar methods & shapes \\
\hline \hline PM & all \\
Jac & all \\
GS & all \\
RGS & all \\
\hline
\end{tabular}

\begin{tabular}{|c|c|}
\hline Block methods & shapes \\
\hline \hline LB & $R_{* \mathcal{B}}$ and $R_{\mathcal{O}_{\mathrm{d}}} \mathcal{T}$ \\
LBR & $R_{* \mathcal{B}}$ and $R_{\mathcal{O}_{\mathrm{d}}} \mathcal{T}$ \\
UB & $R_{* \mathcal{T}}$ \\
UBR & $R_{* \mathcal{T}}$ \\
\hline
\end{tabular}

Figure 7. Numerical Methods Taxonomy. PM is the power method, Jac denotes the Jacobi method, GS and RGS are the Gauss-Seidel and Reverse Gauss-Seidel methods, respectively. All of them can be applied to each transformation of the matrix according to the taxonomy in Figure 3. Among block-methods we have LB and LBR, which can be applied to all lower block triangular matrices, and use GS or RGS to solve each diagonal block. Similarly, UB and UBR refer to the upper block triangular matrices.

In order to have diagonal blocks of smaller size and split the problem into smaller subproblems, we investigate other permutations that further exploit the reducibility of the matrix. Let $\mathcal{J}$ denote the reverse permutation, that is, the permutation which assigns the index $n-i$ to the $i$ th node. To some of the shapes in Figure 4, we can apply another $\mathcal{B}$ operation, sometimes after a reversion of the matrix with the operator $\mathcal{J}$. We obtain the shapes in Figure 6.

Note that the smallest size of the largest diagonal block is achieved for $R_{\mathcal{O}_{\mathrm{a}} \mathcal{B} \mathcal{T} \mathcal{J B}}$. The size of the largest component in $R_{\mathcal{O}_{\mathrm{a}} \mathcal{B} \mathcal{T} \mathcal{J B}}$ is something more than 13 million, while for the permutations in Figure 3, which uses only a BFS, we were able to reach a size of around 17 million.

We adopted ad hoc numerical methods for dealing with the different shapes of the matrices in Figures 4 and 6. In particular, the power method and the Jacobi iterations are compared with the Gauss-Seidel and the Reverse GaussSeidel methods. We recall that the Gauss-Seidel method computes $y_{i}^{(k+1)}$, the $i$ th entry of the vector at the $(k+1)$ th iteration step as a linear combination of $y_{j}^{(k+1)}$ for $j=1, \ldots, i-1$ and of $y_{j}^{(k)}$ for $j=i+1, \ldots, n$. On the contrary, the Reverse Gauss-Seidel method computes the entries of the vector $\mathbf{y}^{(k+1)}$ bottomup, that is, it computes $y_{i}^{(k+1)}$ for $i=n, \ldots, 1$ as a linear combination of $y_{j}^{(k+1)}$ for $j=n, \ldots, i+1$ and of $y_{j}^{(k)}$ for $j=1, \ldots, i-1$. Note that $R_{\mathcal{O}_{\mathrm{d}} \mathcal{T}}=J R_{\mathcal{O}_{\mathrm{a}} \mathcal{T}} J^{T}$ and $R_{\mathcal{I}_{\mathrm{d}} \mathcal{T}}=J R_{\mathcal{I}_{\mathrm{a}} \mathcal{T}} J^{T}$, where $J$ is the anti-diagonal matrix, that is, $J_{i j}=1$ iff $i+j=n+1$. This means that applying Gauss-Seidel to $R_{\mathcal{O}_{\mathrm{d}} \mathcal{T}}\left(R_{\mathcal{I}_{\mathrm{d}} \mathcal{T}}\right)$ is the same as applying Reverse Gauss-Seidel to $R_{\mathcal{O}_{\mathrm{a}} \mathcal{T}}\left(R_{\mathcal{I}_{\mathrm{a}} \mathcal{T}}\right)$.

The shapes of some matrices in Figures 4 and 6 encourage one to exploit the matrix reducibility by experimenting with block methods. Moreover, the matrix $R_{\mathcal{O}_{\mathrm{d}} \mathcal{T}}$ is also lower block triangular, since it separates nondangling nodes from dangling nodes. This matrix is a particular case of the one considered in Equation (5.1). 
As solving methods for the diagonal block systems, we tested both the GaussSeidel and Reverse Gauss-Seidel methods. We denote by LB and UB the methods obtained using Gauss-Seidel as the solver of the diagonal blocks on lower or upper block structures, respectively. LBR and UBR use instead Reverse Gauss-Seidel to solve the diagonal linear systems. Summing up, we report the taxonomy of solution strategies in Figure 7. In Section 6 we report the experimental results obtained by applying each method in Figure 7 to all the suitable matrices in Figure 4 and in Figure 6.

\section{Experimental Results}

We tested the approaches discussed in previous sections using a web graphs obtained by a crawling of 24 million web pages with about 100 million hyperlinks and containing approximately 3 million dangling nodes. This data set was donated to us by the Nutch project (see http://www.nutch.org/). We run our experiments on a PC with a Pentium IV $3 \mathrm{GHz}, 2.0 \mathrm{~GB}$ of memory and $512 \mathrm{~Kb}$ of L2 cache. A stopping criterion of $10^{-7}$ is imposed on the absolute difference between the vectors computed in two successive iterations. In order to have a

\begin{tabular}{|c|c|c|c||c|c|}
\hline Name & PM & GS & RGS & LB/UB & LBR/UBR \\
\hline $\mathcal{T}$ & 345433093152 & 17741995792 & 18182039194 & --- & --- \\
\hline $\mathcal{O}_{\mathrm{d}} \mathcal{T}$ & 293433093152 & 15442082585 & 147719957104 & 14511968096 & 13971886092 \\
\hline $\mathcal{I}_{\mathrm{d}} \mathcal{T}$ & 331533309153 & 18402125998 & 17351995792 & --- & --- \\
\hline \hline $\mathcal{T} \mathcal{B}$ & 138632876151 & 60621910101 & 5191843985 & 40116953100 & 3591505382 \\
\hline $\mathcal{O}_{\mathrm{d}} \mathcal{T} \mathcal{B}$ & 138333093152 & 5822147699 & 5051843985 & 3921748697 & 3691596885 \\
\hline $\mathcal{O}_{\mathrm{a}} \mathcal{T} \mathcal{B}$ & 135332876151 & 61023645109 & 4401692078 & 42418856106 & $\mathbf{3 1 5} \mathbf{1 3 7 8 9} \mathbf{7 5}$ \\
\hline $\mathcal{I}_{\mathrm{d}} \mathcal{T} \mathcal{B}$ & 136133309153 & 5612125998 & 5111909088 & 3851719698 & 3801641487 \\
\hline $\mathcal{I}_{\mathrm{a}} \mathcal{T} \mathcal{B}$ & 139232876151 & 61922343105 & $\mathbf{4 5 0} \mathbf{1 6 2 7 0} \mathbf{7 5}$ & 40017972100 & 3141390575 \\
\hline $\mathcal{B P T}$ & 139433093152 & 5221843985 & 64022560104 & 3791554585 & 47919003104 \\
\hline $\mathcal{O}_{\mathrm{d}} \mathcal{B \mathcal { T }}$ & 134133309153 & 5071887387 & 57921693100 & 3981593787 & 46618312100 \\
\hline $\mathcal{O}_{\mathrm{a}} \mathcal{B \mathcal { T }}$ & 151133093152 & 5911887387 & 68021693100 & 3571512887 & 41317387100 \\
\hline $\mathcal{I}_{\mathrm{d}} \mathcal{B \mathcal { T }}$ & 140833093152 & 5541930689 & 62621693100 & 3971607588 & 45018265100 \\
\hline $\mathcal{I}_{\mathrm{a}} \mathcal{B \mathcal { T }}$ & 135133093152 & 4971843985 & 57521693100 & 3861556485 & 44718310100 \\
\hline
\end{tabular}

Figure 8. Experimental Results: The columns list the numerical methods analyzed, and the rows describe some of the permutations applied to the matrix $R$. Each cell represents the running time in seconds, the number of Mflops, and the number of iterations taken by the solving methods. Note that the results in the last two columns account either for the cost of the LB and LBR methods, applied to lower block triangular matrices, or for the cost of the UB and UBR methods, applied to upper block triangular matrices. In bold we highlight our best results in terms of Mflops for scalar and block methods. 
fair comparison for the power method, the tolerance has been changed "a posteriori" to obtain the same absolute error of the other methods. In fact, in the case of the power method, the PageRank vector has 1-norm equal to one, while for the other methods we do not scale the vector at each step.

In Figure 8 we report the running time in seconds, the Mflops, and the number of iterations for each combination of the solving and reordering methods described in Figure 7 on the matrices of Figure 4. We believe that the number of iterations and the number of floating point iterations of a method are more fair measures than the running time in seconds, which is more implementationdependent. However, the running time is the only factor accounting for an increase in data-locality when a permutation of the matrix does not change the number of iterations.

Some cells of Figure 8 are empty since there are methods suitable only on particular shapes. Moreover, in Figure 8 the results in the last two columns are relative to LB and LBR methods for lower block triangular matrices and UB or UBR for upper block triangular matrices. We do not report in the table the behavior of the Jac method, since it always has worse performance than the GS method. However, the measure of the gain using GS rather than Jac can be obtained from Figure 10. Since the diagonal entries of $R$ are equal to 1, the Jacobi method is essentially equivalent to the power method. In our case, the only difference is that Jac is applied to $R$ while PM works on $\widehat{P}$, which incorporates the rank-one modification accounting for dangling nodes. Although we implemented PM using the optimizations suggested in [Page et al. 98, Haveliwala 99], this method requires a slightly greater number of operations. Using the results of Theorem 4.2, we get a reduction in Mflops of about 3\%. For the increased data locality, the running time of Jac benefits from matrix reordering, and we have a reduction of up to $23 \%$ over the power iterations.

We now compare the proposed methods versus PM applied to the original matrix since this is the common solving method to compute PageRank vector. Other comparisons can be obtained from Figure 8. As one can expect, the use of GS and RGS on the original matrix already accounts for a reduction of about $40 \%$ in the number of Mflops and of about $48 \%$ in running time. These improvements are striking when the system matrix is permuted. The best performance of scalar methods is obtained using the $\mathcal{I}_{\mathrm{a}} \mathcal{T} \mathcal{B}$ combination of permutations on the RGS method. This yields a Mflop reduction of $51 \%$ with respect to PM and a further reduction of $18 \%$ with respect to GS, both applied to the full matrix. The running time is reduced of $87 \%$.

The common intuition is that the Gauss-Seidel method behaves better on a quasi-lower triangular while Reverse Gauss-Seidel is faster when applied to quasiupper triangular matrices. However, in this case the intuition turns out to be 


\begin{tabular}{|c|c|c|c|c|}
\hline Name & GS & RGS & LB/UB & LBR/UBR \\
\hline$\overline{\mathcal{T} \mathcal{B J \mathcal { B }}}$ & 5652147699 & 4881843985 & 4021753499 & 3271398076 \\
\hline $\mathcal{O}_{\mathrm{d}} \mathcal{T} \mathcal{B} \mathcal{J B}$ & 61323645109 & 4451692078 & 42618853106 & 3181379076 \\
\hline $\mathcal{O}_{\mathrm{a}} \mathcal{T} \mathcal{B} \mathcal{J} \mathcal{B}$ & 5472147699 & 4781865686 & 3901789199 & 3621619386 \\
\hline $\mathcal{I}_{\mathrm{d}} \mathcal{T} \mathcal{B} \mathcal{J B}$ & 56822127102 & 4471735480 & 40718458100 & 3211432678 \\
\hline $\mathcal{I}_{\mathrm{a}} \mathcal{T} \mathcal{B} \mathcal{J B}$ & 5472147699 & 4871887387 & 3751717599 & 3681638987 \\
\hline $\mathcal{B} \mathcal{T} \mathcal{J} \mathcal{B}$ & 60522560104 & 4431648676 & 33315905101 & $\begin{array}{llll}246 & 11617 & 73\end{array}$ \\
\hline $\mathcal{O}_{\mathrm{d}} \mathcal{B} \mathcal{T} \mathcal{J} \mathcal{B}$ & 57722560104 & 4151627075 & 33615915101 & 2521177973 \\
\hline $\mathcal{O}_{\mathrm{a}} \mathcal{B} \mathcal{T} \mathcal{J} \mathcal{B}$ & 65521693100 & 5441800583 & 3651589699 & 2801223182 \\
\hline $\mathcal{I}_{\mathrm{d}} \mathcal{B} \mathcal{T} \mathcal{J B}$ & 62322560104 & 4471627075 & 32815896101 & 2501192174 \\
\hline $\mathcal{I}_{\mathrm{a}} \mathcal{B} \mathcal{T} \mathcal{J} \mathcal{B}$ & 55321693100 & 4151627075 & 3281591999 & 2451162472 \\
\hline $\mathcal{B T} \mathcal{B}$ & 5102125998 & 4831995792 & 3821735498 & 3701641091 \\
\hline $\mathcal{O}_{\mathrm{d}} \mathcal{B} \mathcal{T} \mathcal{B}$ & 5192147699 & 4871995792 & 3831680098 & 3761640391 \\
\hline $\mathcal{O}_{\mathrm{a}} \mathcal{B} \mathcal{T} \mathcal{B}$ & 5512125998 & 5221995792 & 3701716898 & 3671640491 \\
\hline $\mathcal{I}_{\mathrm{d}} \mathcal{B T} \mathcal{\mathcal { T }}$ & 5152147699 & 4851995792 & 3681699698 & 3691641091 \\
\hline $\mathcal{I}_{\mathrm{a}} \mathcal{B \mathcal { T }} \mathcal{B}$ & 5092125998 & 4801995792 & 3661679098 & 3661639691 \\
\hline $\mathcal{B} \mathcal{J B T}$ & 5292017493 & 57021693100 & 4131681392 & 45218286100 \\
\hline $\mathcal{O}_{\mathrm{d}} \mathcal{B} \mathcal{J} \mathcal{B} \mathcal{T}$ & 4911865686 & 56621693100 & 3851554585 & 45018281100 \\
\hline $\mathcal{O}_{\mathrm{a}} \mathcal{B} \mathcal{J} \mathcal{B} \mathcal{T}$ & 5011909088 & 58822560104 & 3991610588 & 46418841103 \\
\hline $\mathcal{I}_{\mathrm{d}} \mathcal{B} \mathcal{J} \mathcal{B} \mathcal{T}$ & 4861843985 & 56321693100 & 3871556585 & 44918310100 \\
\hline $\mathcal{I}_{\mathrm{a}} \mathcal{B} \mathcal{J} \mathcal{B} \mathcal{T}$ & 5041930689 & 56521693100 & 3981608188 & 45218269100 \\
\hline
\end{tabular}

Figure 9. Experimental results on the shapes in Figure 6. The columns list the numerical methods analyzed and the rows describe the permutations applied to the matrix $R$. Each cell represents the running time in seconds, the number of Mflops, and the number of iterations taken by the solving methods. In bold we highlight our best results in terms of Mflops for scalar and block methods. We omit the values obtained with the Jacobi method since they are almost unaffected by matrix permutations and can be found in Figure 8 .

misleading. In fact, for our web matrix RGS works better on lower block triangular matrices and GS works better on quasi-upper triangular matrices. Even better results are obtained using block methods. LB applied to $R_{\mathcal{O}_{\mathrm{d}} \mathcal{T}}$ achieves a reduction of $41 \%$ in Mflops with respect to PM. Adopting this solving method, we explore just the matrix reducibility due to dangling nodes as in equation (5.1). The best result is obtained for the $\mathcal{O}_{\mathrm{a}} \mathcal{T} \mathcal{B}$ permutation when the LBR solving method is applied. In this case, we have a reduction of $58 \%$ in Mflops and of $90 \%$ in the running time. This means that our solving algorithm computes the PageRank vector in about a tenth of the running time and with less than half the operations of the power method.

We applied each of the methods in Figure 7 also on the shapes in Figure 6, obtained with two BFS visits. The results are in Figure 9. From Figure 9 we see that we have a further gain when the matrix is split into a greater number of blocks. In fact, we have a reduction up to $92 \%$ on the running time and up to $65 \%$ in terms of Mfops over the power method.

The results given in Figures 8 and 9 do not take into account the effort spent in reordering the matrix. However, the most costly reordering scheme is the BFS 


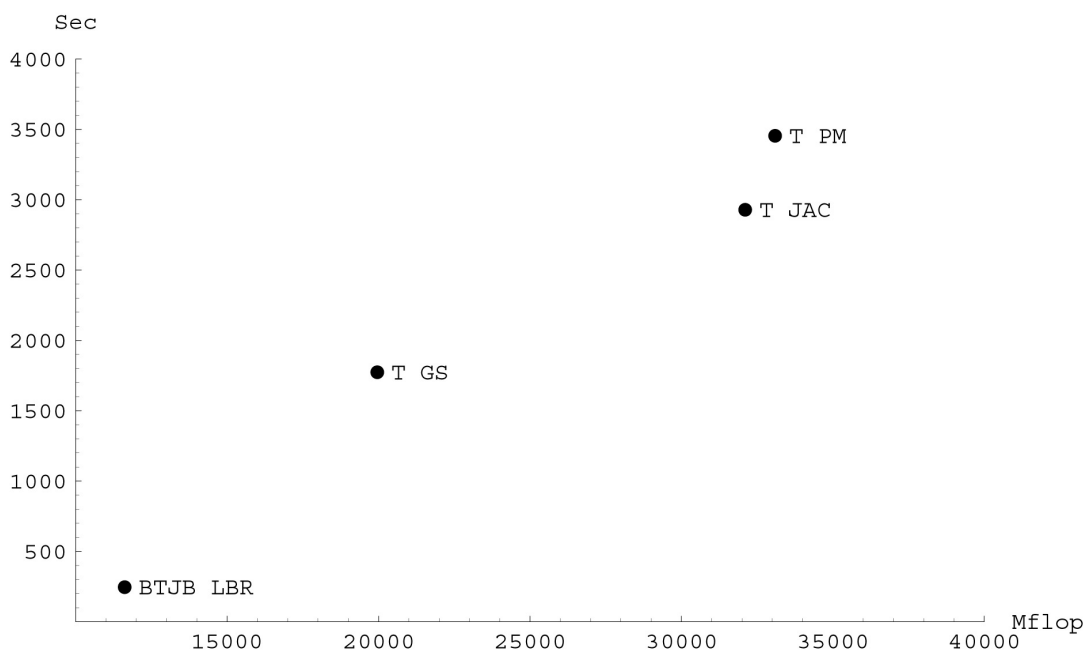

Figure 10. A plot of some results from Figures 8 and 9 with the number of Mflops on the $x$-axis and the running time in seconds on the $y$-axis. Each point is labeled with the permutation applied and the solving method used. The power, Jacobi, and Gauss-Seidel methods applying any permutation are compared with the best result.

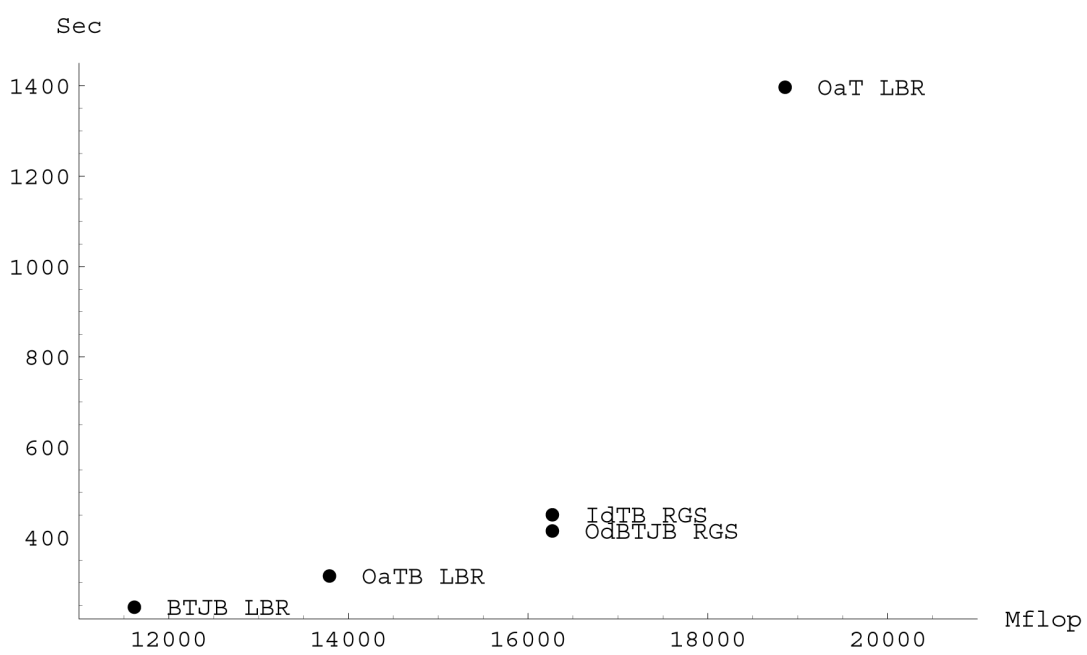

Figure II. A plot of the more interesting results from Figures 8 and 9 with the number of Mflops on the $x$-axis and the running time in seconds on the $y$-axis. Each point is labeled with the permutation applied and the solving method used. The best performance of a method, which permutes the node in separating them into dangling and nondangling without applying any BFS visit, is plotted with the best block and scalar methods that use one or two BFS visits. 
visit of the web graph, which can be efficiently implemented in semi-external memory as reported in [Buchsbaum et al. 00, Mehlhorn and Meyer 02]. The running time spent on doing the BFS are comparable to those reported by Broder et al. in [Broder et al. 00], where less than four minutes are taken on a web graph with 100 million nodes; it is, however, largely repaid by the speedup achieved on the solving methods. Moreover, in the case of personalized PageRank, the permutations can be applied only once and reused for all personalized vectors $\mathbf{v}$. An intuitive picture of the gain obtained by combining permutation strategies with the scalar and block solving methods is shown in Figures 10 and 11.

\section{Conclusion}

The ever-growing size of the web graph implies that the value and the importance of fast methods for web ranking are going to rise in the future. Moreover, the even-growing interest toward personalized PageRank justifies an effort in "preprocessing" the web graph matrix in order to compute faster the many PageRank vectors needed.

The problem of PageRank computation can easily be viewed as a dense linear system. We showed how to handle the density of this matrix by transforming the original problem into one that uses a matrix as sparse as the web graph itself. On the contrary to what was done by Arasu et al. in [Arasu et al. 02], we achieved this result without altering the original model. This result allows one to efficiently consider the PageRank computation as a sparse linear system, an alternative to the eigenpair interpretation.

Dealing with a sparse linear system opens the way to exploiting the reducibility of the web matrix by composing opportunely some permutation strategies to speed up the PageRank computation. We showed that permuting the web matrix according to a combination of in-degrees or out-degrees and sorting the pages following the order of the BFS visit can effectively increase data locality and reduce the running time when used in conjunction with a numerical method such as lower block solvers.

Our best result achieves a reduction of $65 \%$ in Mflops and of $92 \%$ in terms of the seconds required compared to the power method commonly used to compute the PageRank. This means that our solving algorithm requires almost a tenth of the time and much less than half of the Mflops used by the power method. The previous improvement over the power method is due to Lee et al. in [Lee et al. 03] where a reduction of $80 \%$ in time is achieved on a data set of roughly 400,000 nodes. In light of the experimental results, our approach for speeding 
up PageRank computation appears to be very promising especially when dealing with personalized PageRank.

Acknowledgements. Work for this paper was partially supported by the GNCS-INDAM Project: "Problematiche Numeriche nel WEB." The second author was partially supported by the Italian Registry of ccTLD.it.

We thank Daniel Olmedilla of Learning Lab Lower Saxony, Doug Cutting and Ben Lutch of the Nutch Organization, and Sriram Raghavan and Gary Wesley of Stanford University. They provided us with some web graphs and a nice web crawler. We thank Luigi Laura and Stefano Millozzi for their COSIN library. We also thank Paolo Ferragina for useful discussions and suggestions.

\section{References}

[Arasu et al. 02] A. Arasu, J. Novak, A. Tomkins, and J. Tomlin. "PageRank Computation and the Structure of the Web: Experiments and Algorithms." In The Eleventh International World Wide Web Conference Poster Proceedings. Available from World Wide Web ( http://www.ra.ethz.ch/CDstore/www2002/poster/ 173.pdf), 2002.

[Bianchini et al. 03] M. Bianchini, M. Gori, and F. Scarselli. "Inside PageRank." ACM Trans. on Internet Technology 5 (2004), 92-128.

[Boldi and Vigna 04] P. Boldi and S. Vigna. "WebGraph Framework I: Compression Techniques." In Proceedings of the 13th International Conference on World Wide Web, pp. 595-602. New York: ACM Press, 2004.

[Brin and Page 98] S. Brin and L. Page. "The Anatomy of a Large-Scale Hypertextual Web Search Engine." Computer Networks and ISDN Systems 30:1-7 (1998), 107117.

[Broder et al. 00] A. Z. Broder, R. Kumar, F. Maghoul, P. Raghavan, S. Rajagopalan, R. Stata, A. Tomkins, and J. L. Wiener. "Graph Structure in the Web." Computer Networks 33 (2000), 309-320.

[Buchsbaum et al. 00] A. L. Buchsbaum, M. Goldwasser, S. Venkatasubramanian, and J. Westbrook. "On External Memory Graph Traversal." In Proceedings of the Eleventh Annual ACM-SIAM Symposium on Discrete Algorithms, pp. 859-860. Philadelphia: SIAM, 2000.

[Chen et al. 02] Y. Chen, Q. Gan, and T. Suel. "I/O-efficient Techniques for Computing PageRank." In Proceedings of the Eleventh International Conference on Information and Knowledge Management, pp. 549-557. New York: ACM Press, 2002.

[Cho and Roy 04] J. Cho and S. Roy. "Impact of Web search engines on page popularity." In Proceedings of the 13th International Conference on World Wide Web, pp. 20-29. New York: ACM Press, 2004.

[Cuthill and McKee 69] E. Cuthill and J. McKee. "Reducing the Bandwidth of Sparse Symmetric Matrices." In Proceedings of the 1969 24th National Conference, pp. 157-172. New York: ACM Press, 1969. 
[Douglas et al. 00] C. Douglas, J. Hu, M. Iskandarani, M. Kowarschik, U. Rüde, and C. Weiss. "Maximizing Cache Memory Usage for Multigrid Algorithms." In Multiphase Flows and Transport in Porous Media: State of the Art, edited by Z. Chen et al., pp. 124-137. Lecture Notes in Physics 552. Berlin: Springer, 2000.

[Eiron et al. 04] N. Eiron, S. McCurley, and J. A. Tomlin. "Ranking the Web Frontier." In Proceedings of the 13th International Conference on World Wide Web, pp. 390318. New York: ACM Press, 2004.

[Golub and Van Loan 96] G. H. Golub and C. F. Van Loan. Matrix Computations, Third Edition. Baltimore: The John Hopkins University Press, 1996.

[Haveliwala 99] T. Haveliwala. "Efficient Computation of PageRank." Technical report, Stanford University, 1999.

[Haveliwala 02] T. Haveliwala. "Topic-Sensitive PageRank." In Proceedings of the 11th International Conference on World Wide Web, pp. 517-526. New York: ACM Press, 2002.

[Haveliwala et al. 03] T. Haveliwala, S. Kamvar, and G. Jeh. "An Analytical Comparison of Approaches to Personalizing PageRank." Technical report, Stanford University, 2003.

[Jeh and Widom 03] G. Jeh and J. Widom. "Scaling Personalized Web Search." In Proceedings of the 12th International Conference on World Wide Web, pp. 271279. New York: ACM Press, 2003.

[Kamvar and Haveliwala 03] S. Kamvar and T. Haveliwala. "The Condition Number of the PageRank Problem." Technical report, Stanford University, 2003.

[Kamvar et al. 03a] S. Kamvar, T. Haveliwala, C. Manning, and G. Golub. "Extrapolation Methods for Accelerating PageRank Computations." In Proceedings of the 12th International Conference on World Wide Web, pp. 261-270. New York: ACM Press, 2003.

[Kamvar et al. 03b] S. D. Kamvar, T. H. Haveliwala, C. Manning, and G. H. Golub. "Exploiting the Block Structure of the Web for Computing PageRank." Technical report, Stanford University, 2003.

[Langville and Meyer 04] A. N. Langville and C. D. Meyer. "Deeper Inside PageRank." Internet Mathematics 1:3 (2004), 335-400.

[Lee et al. 03] C. P. Lee, G. H. Golub, and S. A. Zenios. "A Fast Two-Stage Algorithm for Computing PageRank." Technical report, Stanford University, 2003.

[Mehlhorn and Meyer 02] K. Mehlhorn and U. Meyer. "External-Memory Breadthfirst Search with Sublinear I/O." In Algorithms-ESA 2002: 10th Annual European Symposium, Rome, Italy, September 17-21, 2002, Proceedings, pp. 723-735. Lecture Notes in Computer Science 2461. Berlin: Springer, 2002.

[Page et al. 98] L. Page, S. Brin, R. Motwani, and T. Winograd. "The PageRank Citation Ranking: Bringing Order to the Web." Technical report, Stanford University, 1998.

[Stewart 95] W. S. Stewart. Introduction to the Numerical Solution of Markov Chains. Princeton, NJ: Princeton University Press, 1995. 
[Varga 62] R. S. Varga. Matrix Iterative Analysis. Englewood Cliffs, NJ: Prentice-Hall, 1962.

[Zhang et al. 04] H. Zhang and A. Goel and R. Govindan and K. Mason and B. Van Roy. "Making Eigenvector-Based Reputation System Robust to Collusion." In Algorithms and Models for the Web-Graph: Third International Workshop, WAW 2004, Rome, Italy, October 16, 2004, Proceedings, pp. 92-104. Lecture Notes in Computer Science 3243. Berlin: Springer, 2004.

Gianna M. Del Corso, Dipartimento di Informatica, Universitá di Pisa, Largo Ponetecorvo 3, 56127 Pisa, Italy (delcorso@di.unipi.it)

Antonio Gullí, Dipartimento di Informatica, Universitá di Pisa, Largo Ponetecorvo 3, 56127 Pisa, Italy (gulli@di.unipi.it)

Francesco Romani, Dipartimento di Informatica, Universitá di Pisa, Largo Ponetecorvo 3, 56127 Pisa, Italy (romani@di.unipi.it)

Received November 1, 2004; accepted August 31, 2005. 